\title{
Lessons to learn with the methicillin-resistant Staphylococcus aureus control in Norway
}

\begin{abstract}
The prevalence of methicillin-resistant Staphylococcus aureus (MRSA) in Norway is low, compared to other European and American countries. The health system includes mandatory case reporting and has written guidelines for prevention and control. This communication describes the national public policies related to MRSA obtained from documents and academic experience gained during a doctoral fellowship in Oslo, Norway. The painstaking procedures used for investigating suspected cases, including health professionals, decolonization and case monitoring, could be important tools to be used by countries with a high prevalence of MRSA.
\end{abstract}

Keywords: Staphylococcus aureus, resistance to methicillin, hospital infection epidemiological surveillance

\section{INTRODUCTION}

The prevalence rates of methicillin-resistant Staphylococcus aureus (MRSA) as an agent of nosocomial infection present relevant differences between American and European countries, with rates above $40 \%$, when compared to Scandinavian countries, where the rates are below $1 \%,{ }^{1}$ including Norway. In this country, the government prioritizes investments in health, education and public services. Cases of infection or colonization by MRSA are subject to mandatory reporting and severe infections are uncommon. ${ }^{2}$

As part of a $\mathrm{PhD}$ course and studying this issue, we carried out a fellowship in Oslo from October 1, 2007 to January 31, 2008, supported by CAPES/ME, in the Ullevall University Hospital under the supervision of Prof. Dr. Bjorg Marit Andersen. This hospital has 1,200 beds and 6,200 staff, and serves a population of 150,000. Its protocol for MRSA screening and decolonization for both patients and health professionals differs in part from the national recommendations due to its greater rigor. ${ }^{3}$

This article aims to report both Norwegian public policy and Ullevål's institutional policy for the prevention and control of MRSA, based on official documents ${ }^{3,4}$ and our academic experience.

\section{PREVENTION AND CONTROL OF MRSA}

All health institutions should maintain preventive measures and, in accordance with national guidelines, follow recommendations for the use of antibiotics and the reporting of cases of MRSA. The guidelines include measures to be taken when investigating patients originating from outside the Netherlands or Scandinavia and health professionals suspected of colonization.

\section{Measures for hospitals}

Patients suspected of colonization or infection by MRSA are investigated and kept in isolation (private room with negative pressure and a private bathroom). In the Ullevål Hospital, the following body sites are investigated, using a swab moistened with saline solution: the inner portion and distal nasal vestibule, where the swab should be circled three times in each nostril, and the throat, including the uvula and both tonsils: both hands (between fingers and under nails) and wrists; and the genital region (the swab should be passed three times between the scrotum/vulva towards the anus). In the case of eczema, a wound or other injuries, and drains, samples should be taken. In the case of catheters, the swab should be circled
Authors

Elaine Drehmer de Almeida $\mathrm{Cruz}^{1}$

Fabiana Cristina Pimenta ${ }^{2}$ Bjorg Marit Andersen ${ }^{3}$ Elucir Gir ${ }^{4}$

${ }^{1} \mathrm{PhD}$; Associate Professor, Member of Multiprofissional Research Group on Adult Health (GEMSA), Nursing Departament, Universidade Federal do Paraná, Brazil ${ }^{2} \mathrm{PhD}$; Researcher, Centers for Disease Control and Prevention, Atlanta, United States

${ }^{3} \mathrm{PhD}$; Chief, Hospital Hygiene Service, Hospital Ullevål, University of Oslo, Norway ${ }^{4} \mathrm{PhD}$; Full Professor, Nursing School, Universidade de São Paulo, Ribeirão Preto, São Paulo, Brazil

Submitted on: $05 / 17 / 2010$ Approved on: 09/20/2010

Correspondence to: Elaine Drehmer de Almeida Cruz

Universidade Federal do Paraná

Rua Lothário Meissner, 632 Bloco Didático II

Setor de Ciências da Saúde Campus Botânico 80210-170 - Curitiba, PR Brazil

elainedrehmer@yahoo.com.br

Financial Support: Article related to a doctorate internship carried out as part of a sandwich course at the University of Oslo, Norway, supported by CAPES (process \#2204/07). Interunits Program, Ribeirão Preto Nursing School, Universidade de São Paulo.

We declare no conflict of interest. 
three times around the insertion site. The guidelines recommend collection of two sets, with an interval of one to two hours, for the nasal vestibule, the perineum, injured skin tissue, pus, catheters and artificial openings.

Health professionals should wear a surgical mask, apron and gloves when in direct contact with suspected cases. Should a positive case be detected unexpectedly, patients and healthcare professionals who have been in contact with that person are also to be considered as suspected carriers.

Infected or colonized patients, following treatment or decolonization, have their results monitored and receive written advice.

\section{Measures for long-stay institutions}

MRSA is a problem on the rise in primary care institutions and also in nursing homes, which are also termed "longterm care facilities" (LTCF), for mostly being long-stay facilities. One concern is that, while preventive measures in LTCF should be the same as those recommended for hospitals, the former are residences where keeping MRSA carriers in isolation is not feasible. With this in view, experiments have been carried out with the objective of altering behavior and procedures, in order to render viable control and prevention. The routine for eradication of MRSA is the same as those required in hospital. For persistent carriers, a private bathroom is recommended, although in LTCF a negative pressure air system is not required.

\section{Measures for home care}

The increase of MRSA carriers in the community carrying a specific genotype (SCCmecIV) indicates that a MRSA clone detected in hospitals has spread to the community, with some modifications. Considering the increasing possibility of public service users of home care (widely used in Norway) being carriers of MRSA, all homecare staff should wear gloves, mask and apron when in direct contact with patients or surfaces. The principles of suspicion of colonization, criteria for research and independence are the same as for hospitals and LTCF. ${ }^{5}$

\section{Measures for health professionals}

Health professionals who have worked in the past six months in the health service with a history of outbreaks of MRSA, in Norway or abroad, are not allowed to perform professional activities until the results of screening of skin lesions and nasal vestibule (removal of two sets with an interval of two hours) are known and negative. In the case of those with a previous history of contact with an LTCF user in a country where MRSA is endemic, the doctor may be able to initiate activities before knowing culture results.

In Ullevål Hospital, health professionals have to be screened on returning from holidays abroad. This procedure is also applied to those who have been exposed to MRSA in the last 12 months, or those with a previous history of colonization or infection. Until the healthcare professional has con- firmation from the results of screening that he/she is clear of MRSA, when in direct contact with the patient or their care environment he/she should use a face mask, apron and gloves.

Positive results are reported by the laboratory directly to the physician and the Infection Control Office. The physician should inform the patient, family and health professionals involved in their care. He/she should also coordinate control measures, list patients and health grounds and communicate to the laboratory the number of samples required to be processed.

Colonized health professionals cannot perform activities in the health system and must be decolonized and the results followed for a period of six months. At Ullevål Hospital, seven days after decolonization, three new screening cultures are taken at intervals of three to seven days. If the results are negative, MRSA is considered inactive. In the following 3, 6,9 , and 12 months, new screenings will be carried out, and if all the results are negative, the professional is considered likely MRSA negative.

\section{Sanitation measures for MRSA - Ullevål Hospital}

The following measures are recommended for patients during hospitalization and after discharge, and for colonized health professionals; they include physical decolonization and environmental measures in hospital, at home or in LTCF, to be repeated over ten days.

It is recommended to sanitize hands between 10 and 20 times a day with alcoholic chlorhexidine or other antiseptic alcohol, or alternatively with tissues moistened with alcohol solution.

To decolonize, it is recommended to soap the body, while for the hair one should use chlorhexidine detergent for two minutes, rinse with water and then repeat the procedure. Areas between fingers, perineum, navel and hair must be specially cleaned.

Shower cubicle walls should be cleaned with warm water; the towels (separate towels for body and feet), clothes and nightclothes should be washed daily at a temperature between $65^{\circ}$ and $85^{\circ} \mathrm{C}$. Considering the frequent contamination of the environment (ground, bed, door handles and other surfaces) daily disinfection with alcohol $70 \%$ is recommended.

The mouth and throat should be rinsed by gargling with mouthwash with chlorhexidine oral solution or hydrogen peroxide. The toothbrush should be rinsed in running water at a temperature exceeding $65^{\circ} \mathrm{C}$ for one to two minutes or immersed in chlorhexidine alcoholic solution. The application of mupirocin in the nasal mucosa with swabs is recommended, three times a day for ten days.

Personal items such as hairbrushes, sunglasses and contact lenses should be disinfected daily. It is recommended that one should avoid the use of soap bars, jewellery and makeup. Tubes and bottles should be cleaned externally and, where possible, should be of single use. 


\section{FINAL CONSIDERATIONS}

We were able to experience the planning and implementation of MRSA prevention and control measures in Norway and see the rigorous adoption of epidemiological surveillance measures. We emphasize work conditions, in terms of access to personal protection equipment and its quality and quantity, human resources, and also the physical infrastructure for isolation. This last includes: individual rooms with private bathrooms, negative pressure and ideal conditions for hand-cleaning: the availability, inside the patient's bathroom, of thermal disinfection for hygiene utensils: and a means for the removal of contaminated materials.

Another factor is the accountability of health professionals who provide assistance, with emphasis on leadership and medical care, and on the adoption of measures for prevention and control, particularly in the investigation of contacts. These activities are only supervised by the Office of Infection Control, and are not under their responsibility.

Not all LTCF have the necessary infrastructure and human resources, which is perceived as one of the reasons for the occurrence of MRSA outbreaks and high levels of colonization. However, health authorities in this country that prioritizes the health and welfare of its citizens are aware of the problems that need correcting.

We believe that the rigorous policy of identifying and managing cases and suspects is a key factor for the low prevalence of MRSA. Although it might not be possible to fully adopt such policies in other countries in Europe or the Americas, including Brazil, they could serve as inspiration and for reflection on the measures currently used - and on their disappointing results.

\section{REFERENCES}

1. EARSS. European Antimicrobial Resistance Surveillance System. EARSS. Annual report: 2007. Available on: <http://www. rivm.nl/earss/result/Monitoring_reports/Annual_reports. jsp >. Access on: 25 Jul 2011.

2. Folkehelseinstituttet. Norwegian Institute of Public Health. Surveillance of communicable diseases and nosocomial infections in Norway 2006: trends and main prevention strategies - MRSA. Oslo, 2007.

3. Andersen BM, Rasch M, Syversen G. Is an increase of MRSA in Oslo, Norway, associated with changed infection control policy? J Infect 2007; 55(6):531-8.

4. Ullevål Hospital. Ullevål Standard Guideline. Oslo, 2007.

5. Fossum AE, Bukholm G. Increased incidence of methicillinresistant Staphylococcus aureus ST80, novel ST125 and SSCmecIV in the south-eastern part of Norway during a 12-year period. Clin Microb Infect 2006; 12(7):627-33. 